\title{
The effectiveness of European regulation for vehicle noise control
}

\author{
R. de Abrantes, A. L. S. Forcetto \& R. M. Araújo \\ Cetesb, Environmental Agency of Sao Paulo State, Brazil
}

\begin{abstract}
Europe is changing its regulation on the noise emitted by vehicles because the European community realized that noise levels on streets and in buildings are higher than expected. There are many possible reasons for this public health problem, among others: the growth of the vehicle fleet and the inefficiency of the type of approval method.

In response to this problem, the ISO 362-1 has been revised, and now vehicles are tested under less severe conditions but, in the light of the driving habits in the urban centres, experts say this new procedure is more realistic than the previous one.

However, on critical analysis, one can find some points, such as the lack of importance given to the engine cooling system and the way in which urban buses are assessed in the same way as delivery trucks, among other points that may annul the effect of this new European proposal.

Vehicle manufacturers hope that ISO $362-1$ will be adopted by as many countries as possible around the world, in order to reduce development costs, but some weak points may make its acceptance by other countries' governments difficult.

Many countries around the world are facing the same environmental problem faced by Europe, and they need to improve their legislation on vehicle noise control, but some experts are not fully convinced as to the environmental benefits of this new method and tend to adopt other methods, considered more effective, which can be a problem for vehicle manufacturers, due to the final costs of vehicles.

This study seeks to discuss some of these points of possible improvement and show what it is possible to do to arrive at an effective global method to produce
\end{abstract}


good results which will, probably, be of easier acceptance on the part of other countries not belonging to the European Union.

Keywords: vehicle noise source, noise pollution, ISO 362-1, pass-by noise.

\section{Introduction}

The aim of this study is to discuss the effectiveness of the new European regulation on noise emitted by road vehicles and address some points calling for improvement which may produce better results and, if adopted, probably make its acceptance by non-European Union countries easier. This paper does not intend to discuss the subject generically, but rather to focus on the categories M2, M3, N2 and N3 (see Appendix), corresponding to heavy duty vehicles (HDV), i.e. basically, buses and trucks, not just because diesel engines, which usually are used in HDV, emit higher noise levels than petrol engines when compared at the same power and at the same angular speed, but because there are some aspects of the subject which were not taken into consideration, and that without proper consideration and control can make the global results less effective than expected. Light duty vehicles (LDV) will also be discussed, but in order to compare them with the participation of HDV. Quality of test track, tyres and category L, corresponding to motorcycles and three-wheeled vehicles, whose noise participation is considerable, are not considered in this paper.

\section{The methods for pass-by noise control}

In Europe the previous vehicle noise regulation was regulated by ECE R51.02, the tests for which were based on the standard method of ISO 362:1998, the guideline for the implementation of the vehicle pass-by noise test, and that we denominate simply method $\mathrm{A}$ in this paper, for ease of reference $[1,2]$.

The test facility consists of a $20 \mathrm{~m}$ by $20 \mathrm{~m}$ acceleration area with a reference line along its centre, representing the driving direction of the vehicle. The acceleration area and the driving lane $10 \mathrm{~m}$ prior to and after the acceleration area are asphalted, according to the requirements of the ISO 10844 standard, once the quality of the surface can affect the results.

Two microphones are positioned midway of the acceleration area at a distance of $7.5 \mathrm{~m}$ away from the reference line and at a height of $1.2 \mathrm{~m}$. For LDV, the vehicle is driven along the reference line approaching the acceleration area at a constant speed of $50 \mathrm{~km} \cdot \mathrm{h}^{-1} \pm 1 \mathrm{~km} \cdot \mathrm{h}^{-1}$. When the front of the vehicle reaches the acceleration area, it is fully accelerated and the vehicle traverses the acceleration area in the wide open throttle (WOT) condition, i.e. at maximum acceleration. When the rear of the vehicle exits the test area, the run is finished. The maximum A-weighted pass-by noise level is recorded by the two microphones. For the majority of LDV with manual transmission, the pass-by is driven in 2nd and 3rd gears. Four consecutive runs are carried out for each gear. They are averaged for each side and each gear, resulting in the pass-by noise level. The measured passby noise levels of each gear have to be within a range of $2 \mathrm{~dB}(\mathrm{~A})$ [3]. 
During the test, meteorological conditions have to be within certain tolerance ranges and must be monitored. High wind speeds and rain invalidate the test. The only decision criterion of the pass-by noise test is the maximum A-weighted sound pressure level; the sound quality or frequency is not taken into consideration. Thus also, when fan cooling starts during a run, the noise usually increases more than $2 \mathrm{~dB}(\mathrm{~A})$, so measurements taken under these circumstances are not considered.

For HDV the test facility is the same, the main difference relates to the approximation speed as, depending on the power curve of the engine, the target cannot be $50 \mathrm{~km} \cdot \mathrm{h}^{-1} \pm 1 \mathrm{~km} \cdot \mathrm{h}^{-1}$, but a certain speed, where the engine rotation is $3 / 4$ of the rotation of maximum power for vehicles equipped with engines not greater than $225 \mathrm{~kW}$ or half of the rotation of maximum power for vehicles equipped with engines more powerful than $225 \mathrm{~kW}[1,3]$.

Experiments of noise isolation were carried out in cars, using a vehicle shielding technique in order to identify noise sources. The results have shown that the relative level of noise from each source depends upon the position of the vehicle on the test track. At the beginning of the test track, the noise from the air intake system is dominant. In the middle of the track, when the distance of the vehicle from the microphone is the shortest, the powertrain noise is greatest. At the end of the track, the exhaust system noise is dominant [3].

In the process of the development of a vehicle, manufacturers have to ensure compliance with legislation, which has to be verified prior to its market launch. In Europe, gradual noise reductions have been imposed on the noise emission limit, the LDV limit decreased from $82 \mathrm{~dB}(\mathrm{~A})$ in the 80 s to $74 \mathrm{~dB}(\mathrm{~A})$ in the $90 \mathrm{~s}$. This means that noise in urban centres should be reduced by the replacement of the fleet over time or that it would be possible to multiply the vehicle fleet by ten to obtain the same noise level on the streets, but in practical terms the noise has turned out to be much greater than expected $[4,5]$.

Noise has, in fact, been increasing in urban centres, above the level recommendation by $\mathrm{WHO}$, a fact that has not been observed just in Europe but in many countries throughout the world [5]. Noise in urban centres is due to a variety of different sources such as neighbours, sirens, aircraft, railways, and road traffic, among others [6].

Inquiries among city-dwellers in many cities have shown that noise disturbance comes mainly from vehicles and occurs predominantly on thoroughfares and when vehicles accelerate strongly [6].

Road traffic noise represents a burden to people, mainly in urban centres, giving rise to annoyance [3]. Traffic noise has been ranked as the second main environmental stressor. There are strong correlations between noise and cardiovascular diseases and cognitive impairment, sleep disturbance, tinnitus and annoyance. In populations exposed to high noise levels there is a greater risk of high blood pressure and myocardial infarction, which increases exponentially [5].

Sleep disturbance is related to a long list of problems, affecting walking motor performance, memory consolidation, creativity, risk-taking behaviour, signal detection performance and risk of accidents. People subject to high noise 
levels, especially intermittent noise during the night, do not realize the reason for their sleep disturbance but still feel its effects [5].

In vehicles, the causes of noise generation and the subsequent noise source radiation characteristics are, in general, very complex. The main source of the radiation of engine noise lies behind the front axle and is transmitted through the gap between the lower side of the vehicle body and the ground and towards people [3].

There are several causes for the lack of effectiveness of method A: modifications made to used vehicles, ineffective control of production, disregard of noise from the air cooling fan, the use of the same type approval method for vehicles of different applications such as buses and trucks, and finally, as a result, method A is inappropriate for the environmental approval of vehicles [7].

One of the main criticisms regarding the effectiveness of method A is its poor simulation of typical urban vehicle noise produced by the procedure (wide-open throttle, second and third gear). This was aggravated by implementations of ISO 362:1998 which further impacted the poor correlation between real traffic and the reported regulatory results by allowing the use of minimum tread depth tyres. A further reason for the poor simulation of typical urban vehicle noise is the technical development of vehicle engine and transmission technologies that have invalidated some of the original technical assumptions behind ISO 362:1998 [6].

Due to the full acceleration required, the pass-by test is dominated by powertrain noise which masks the contribution of tyre noise. Urban traffic noise is not characterized by full accelerating vehicles, since this situation is infrequent, so in urban centres tyre noise does, in fact, have a greater contribution [6].

In order to understand the problem better, data from 61 vehicles in real urban use were collected. Almost all categories of vehicles (M1, N1, N2, and one N3 up to 19 tons) were driven in some European and Japanese cities and their behaviour recorded. This list includes vehicles of power ranging from $40 \mathrm{~kW}$ to $440 \mathrm{~kW}$. Just one vehicle of category N3 was used and no bus was assessed, despite this small HDV sample, it was considered representative of the European market [6].

So, in the light of the inadequacy of method A, the European Union proposed a new procedure that has made it possible to improve measurement of the level of the noise actually emitted by vehicles in urban traffic and which is more appropriate to the technical developments in vehicle propulsion and transmission technology. This proposal is more effective in the reduction of vehicle noise emission using an improved metric, that, in this paper, for ease of reference, we denominate method B [6].

The European Parliament and the European Council adopted, in 2014, the regulation (EU) n. 540/2014 of the European Parliament and of the Council on the sound level of road vehicles and of replacement silencing systems, emended Directive 2007/46/EC and repealed Directive 70/157/EEC [12].

The procedure defined by method B, maintains some similarities with method A, e.g., it uses the same test facilities. For LDV, vehicles are tested in two situations: a) when accelerating, the target speed is still $50 \mathrm{~km} \cdot \mathrm{h}^{-1}$, but it must 
occur when the front of the vehicle reaches the central line between the two microphones and the acceleration is no longer WOT, but a partial acceleration in order to simulate urban traffic conditions, depending on the ratio between the engine's power and the vehicle's mass, known as Power to Mass Ratio (PMR), and $\mathrm{b}$ ) at a constant-speed of $50 \mathrm{~km} \cdot \mathrm{h}^{-1}$.

The final result for LDV is calculated by a weighted combination of the acceleration test results and the constant speed test results, using a partial power factor, according to the vehicle's PMR.

In HDV, full throttle acceleration has been found to be the typical acceleration in urban traffic, the requirement for this type of approval test is, therefore, carried out under the WOT condition. However, the target vehicle speed is $35 \mathrm{~km} \cdot \mathrm{h}^{-1} \pm 5 \mathrm{~km} . \mathrm{h}^{-1}$, when the vehicle's reference point pass 5 metres beyond the end of the acceleration area and, at that moment, the engine speed must be between $70 \%$ and $74 \%$ of the engine speed that obtain the rated maximum power for vehicles of category M2 with maximum authorized mass exceeding 3,500 kg, and vehicles of category N2. For categories M3 and N3 the engine speed must be between $85 \%$ and $89 \%$ of its rated maximum net power. The reference point for HDV is the front end of the vehicle for front-engine vehicles, or the edge of the engine closest to the front of the vehicle for all other vehicles $[3,4,6]$.

To limit the vehicle acceleration for categories N2 and N3 to a realistic value, it is mandatory to add a payload to the vehicle: the total mass of the vehicle to be tested should correspond to $50 \mathrm{~kg} / \mathrm{kW}$, limited to $75 \%$ of the maximum weight allowed on the rear axle.

The measured sound pressure level corresponds to the 90th percentile of the maximum noise emitted during the acceleration phase in urban traffic. The method provides excitation of all significant vehicle noise sources to provide the 90th percentile estimate of a vehicle's noise emission in an urban environment [6].

This method is based on the performance criteria of acceleration and is independent of the vehicle's technology, transmission type, number of transmission gears, and type of engine. These performance criteria make this method applicable to current and future vehicles, including adaptive automatic transmissions, hybrid vehicles, electric vehicles, and fuel cell vehicles [6].

\section{Analysis}

Reading ISO 362-1:2015 one can find concepts that are fully justified used in developing the new procedure for categories M1 and M2 with a maximum authorized mass not exceeding $3,500 \mathrm{~kg}$ and category $\mathrm{N} 1$, but there is no justification for applying the procedure to other vehicle categories, which can hinder its acceptance by some $[4,6]$.

When performing according to method $\mathrm{B}$, vehicles are tested under less demanding conditions than in method A, but considering the normal driving style adopted in urban centres, experts say this new procedure reflects urban driving behaviour more realistically than the previous one [3]. In practical terms, 
the test is done at a lower speed and with lower engine rotation, consequently generating less noise. This fact is compensated for by the noise emission limits that are lower than the previous ones, according to the vehicle's category.

The correlation between method A and method B is very weak for categories M1 and N1. There is a general trend for method B's test results to be somewhat lower [4]. No correlation between the two methods was found for HDV in the literature, so lower emission limits do not necessarily translate into environmental benefits, because in real use on the streets, vehicles approved by method B can emit a higher noise.

Tyre noise is dominant over propulsion noise for speeds higher than $35 \mathrm{~km} \cdot \mathrm{h}^{-1}$ for LDV, especially at constant speeds. The propulsion noise is negligible. At speeds higher than $80 \mathrm{~km} \cdot \mathrm{h}^{-1}$ for LDV, however, in the case of HDV, the contribution of propulsion noise cannot be neglected [8].

To fulfil the demands of method B, the manufacturers, responsible for vehicle noise insulation, tend to be proportionally less demanded in the development of their products, as the less severe the acceleration the greater the proportional contribution of tyre noise. Tyres manufacturers have to meet the obligation to offer more silent tyres to the market, but this demand is difficult to meet in terms of the rubber formula, because of the concomitant demand for safer "green tyres", i.e. low rolling resistance tyres [8]. Considering that a less robust noise shield will be necessary, despite a probable increase in the cost of tyres, it can reduce development costs and consequently the final cost of vehicles [9].

During the research project, a difference of about $12 \mathrm{~dB}(\mathrm{~A})$ was found between the noise emitted by the noisiest and the quietest of common HDV tyres. Little is known about the majority of the mechanisms involved in this application of tyres; it is unclear whether it would be possible to design HDV tyres which produce less noise on common road surfaces and lower noise than the present tyres under highway conditions, so making this demand on HDV tyre manufacturers might be counterproductive $[8,10,11]$.

To be able to develop a broader policy to reduce HDV tyre noise emission in practice it is necessary to gain more insight into the basic fundamentals underlying the mechanisms of noise emission and the effect that tyre characteristics, operating conditions and the environment have on the production of noise [11].

Both methods (A and B) consider vehicles to have four major noise sources: the engine, the air-intake system, the exhaust system and the tyres, but the noise from the air-cooling fan should also be taken into consideration [3]. It is clear that an important noise source has been neglected since the beginning of vehicle noise control, i.e., the air-cooling fan.

The air-cooling fan is part of the cooling system which operates together with the radiator and can function permanently or occasionally by a variety of mechanisms such as viscous coupling, electrical or pneumatic power. Ultimately the principal function of the cooling system is to keep the engine temperature within an appropriate working range, generally around $90^{\circ} \mathrm{C}$. If the engine temperature rises beyond this, the cooling system starts, lowering engine temperature and so avoiding its breaking-down [13]. 
In both methods, the noise emitted by the vehicle in motion is only considered a valid criterion when the differences between the measurements on the same side of the vehicle do not exceed $2.0 \mathrm{~dB}(\mathrm{~A})[1,6]$. So, when the fan of the cooling system turns on, the noise emitted is much higher than $2 \mathrm{~dB}(\mathrm{~A})$, so the reading is not taken into consideration. In practical terms, every time the engine cooling system works during the noise evaluation, the reading is not registered. It is as if the noise from the cooling system were negligible, or as if people were not affected by it, what is an absurd in terms of public health.

In HDV, the fan's diameter is, not rarely, greater than half a metre, and when it is working it increases the vehicle's noise by up to $6 \mathrm{~dB}$, which corresponds to multiplying the noise energy released by four, considering that noise is measured on a logarithmic scale, it as if three other vehicles suddenly appeared each time the cooling system turns on: this noise source cannot, therefore, be neglected.

An evaluation conducted on the streets of the Metropolitan region of Sao Paulo, Brazil, was carried out with urban buses by a method similar to method A and it was observed that of the 75 vehicles assessed, 42 had their cooling systems turned off, the resulting maximum noise average being $80.6 \mathrm{~dB}(\mathrm{~A})$, while $33 \mathrm{had}$ their cooling systems turned on and the maximum noise average was $84.8 \mathrm{~dB}(\mathrm{~A}) ; 94 \%$ of the buses with their cooling systems working produced noise higher than the Brazilian noise limit allowed for this vehicle category, which is $81 \mathrm{~dB}(\mathrm{~A})$. The results of this evaluation are shown in Figure 1.

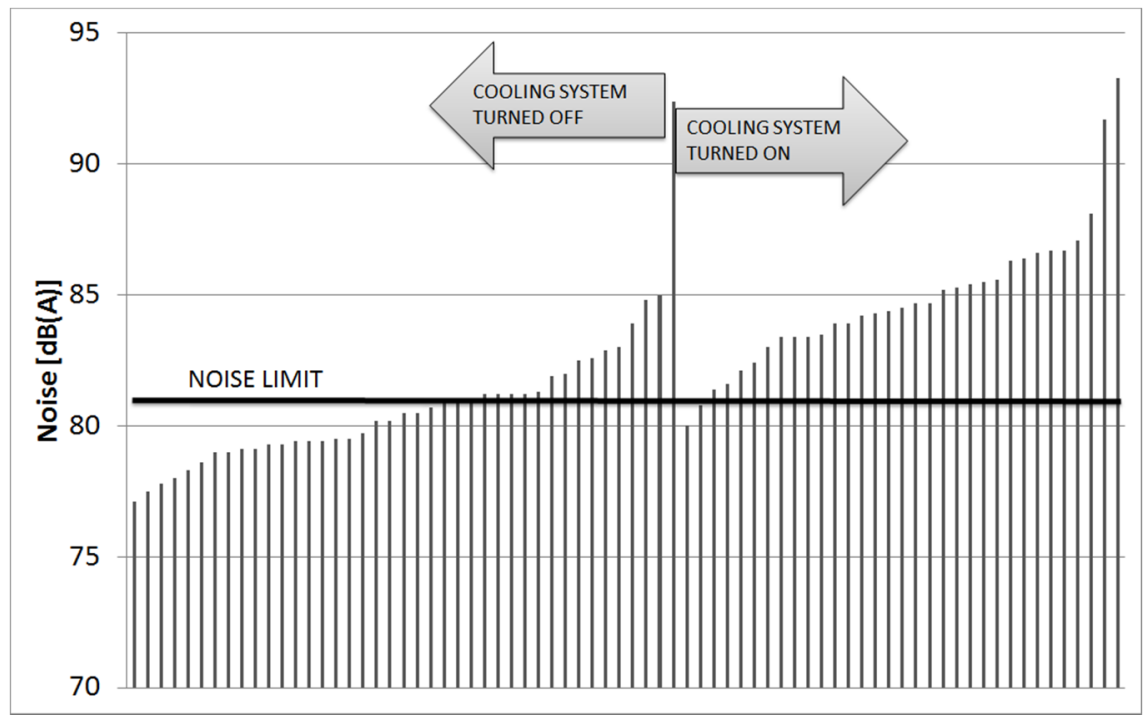

Figure 1: Noise evaluation on the streets of the Metropolitan region of Sao Paulo with 75 urban buses, ordered by cooling system status and noise emitted. 
The HDV fleet's size is significant also, although numerically it is far smaller than the LDV fleet one must keep in mind that the HDV fleet is used generally more than 10 hours a day.

The body of buses is very different from that of trucks. Usually trucks are equipped just with frontal engines, which mean that the cooling of the engine is easier, but buses are much more complex, because there are buses with frontal, middle or rear engines, and apart from that, the chassis can be of just one block, articulated or bi-articulated, which makes the engine cooling more complex.

Moreover, trucks have a very different application to buses, even if one compares small trucks, usually for deliveries in the cities, the application is different. Bus usage has a strong characteristic of stop and go much more severe than the urban trucks that make deliveries. Considering that the requirement of European regulation is the same for both applications, what implicates in vehicle development cannot match real vehicle usage, and so cannot be as effective as expected on the streets.

\section{Final considerations}

There is no doubt that the new regulation represents an advance in vehicle noise control in Europe, and this example can be followed by many countries around the world, as European vehicle manufacturers desire, since development costs can thus be reduced. However, despite experts' efforts, it is clear that some omissions committed in the past have been repeated, which may mean that method B is only partially successful.

To guarantee the success of noise control, it is important to have a clear idea as to how the following subjects should be treated in the future:

- Inspection and maintenance of used vehicles;

- Control of production;

- Noise from engine-cooling system;

- $\quad$ Proper type approval method for each application.

Some modifications in used vehicles can be undertaken along their lives, such as removing the revetment for the isolation of noise, changes in the vehicle body, changes in tailpipe configurations, and so on. It is thus very important that materials used in noise insulation in a vehicle's body be checked periodically, especially for HDVs, in order to keep noise within expected values.

Considering that the environmental licensing process is based on a prototype evaluation, an effective Control of Production $(\mathrm{CoP})$ is necessary to guarantee that all the components used to reduce noise emission be mounted in the vehicles throughout the life of the model. Further CoP programmes must ensure their quality throughout the life of the vehicle model.

Considering that the engine-cooling system can multiply by up to four times the noise energy emitted in HDV, it is not possible to continue to neglect this significant noise source, because the population is being exposed to it daily. The next step in the European regulation should consider noise limits for enginecooling systems. With the knowledge acquired regarding the properties of new materials and aerodynamics, it is possible to obtain good noise insulation without 
any significant impact on the final cost of vehicles by using cheap materials or simply modifying the fan shape, without compromising the effectiveness of the system.

Considering that buses have an application very different from that of trucks, a specific test procedure should be developed for each application, so a specific stop-and-go noise test for urban buses should be considered. The development costs of vehicles will be the same; but the effectiveness will be higher.

Many countries around the world are facing the same environmental problem and need to improve their legislation on noise emitted by vehicles, but some experts are not fully convinced as to the environmental benefits of this new method, and are inclined to adopt other methods, such as housing solutions considered more effective. This may constitute a problem for European vehicle manufacturers in terms of the final costs of vehicles. But if some of these proposals are considered, the European regulation will be improved and the exposure of the European population to noise will be reduced. Then such steps will easily adopted by other countries.

\section{Appendix}

\section{Vehicle categories according to ISO 362-1:2015}

\section{Category L}

Motor vehicles with fewer than four wheels. Note 1 to entry: United Nations Economic Commission for Europe (UNECE) document TRANS/WP.29/78/ Rev.1/Amend.4 (26 April 2005) extended the L category to four-wheeled vehicles as defined by L6 and L 7 .

\section{Category $M$}

Power-driven vehicles having at least four wheels and used for the carriage of passengers.

\section{Category M1}

Vehicles used for the carriage of passengers and comprising no more than eight seats in addition to the driver's seat.

\section{Category M2}

Vehicles used for the carriage of passengers and comprising more than eight seats in addition to the driver's seat and having a maximum mass not exceeding $5,000 \mathrm{~kg}$. Note 1 to entry: In this definition, "maximum mass" is equivalent to "maximum authorized mass" used elsewhere in this part of ISO 362.

\section{Category M3}

Vehicles used for the carriage of passengers and comprising more than eight seats in addition to the driver's seat and having a maximum mass exceeding $5,000 \mathrm{~kg}$. Note 1 to entry: In this definition, "maximum mass" is equivalent to "maximum authorized mass" used elsewhere in this part of ISO 362. 


\section{Category $\mathbf{N}$}

Power-driven vehicles having at least four wheels and used for the carriage of goods.

\section{Category N1}

Vehicles used for the carriage of goods and having a maximum authorized mass not exceeding $3,500 \mathrm{~kg}$.

\section{Category $\mathbf{N 2}$}

Vehicles used for the carriage of goods and having a maximum authorized mass exceeding $3,500 \mathrm{~kg}$ but not exceeding $12,000 \mathrm{~kg}$.

\section{Category N3}

Vehicles used for the carriage of goods and having a maximum authorized mass exceeding $12,000 \mathrm{~kg}$.

\section{References}

[1] United Nations Economic Commission for Europe. Regulation No. 51: Uniform provisions concerning the approval of motor vehicles having at least four wheels with regard to their noise emissions. E/ECE/324/Rev.1/Add.50/Rev.2-E/ECE/TRANS/505/Rev.1/Add.50/Rev. $2,2011$.

[2] International Standard Organization (ISO). ISO 362: Measurement of noise emitted by accelerating road vehicles - engineering method - Part 1: $\mathrm{M}$ and N categories. London: British Standards Institution; 2007.

[3] Braun et al. Noise source characteristics in the ISO 362 vehicle pass-by noise test: literature review. Applied Acoustics, 74 (2013), pp.1241-1265, 2013.

[4] International Organization of Motor Vehicle Manufacturers (OICA). Development of a New Global Type Approval Test Procedure for Motor Vehicles With Regard to Their Noise Emissions. International Symposium of automotive Engineering. Automotive engineering Association, Symposium proceedings, 2007.

[5] World Health Organization (WHO). Burden of disease from environmental noise: Quantification of healthy life years lost in Europe. WHO Regional Office for Europe: Copenhagen, p. 126, 2011.

[6] International Standard Organization (ISO). ISO 362: Measurement of noise emitted by accelerating road vehicles - engineering method - Part 1: $\mathrm{M}$ and $\mathrm{N}$ categories. London: British Standards Institution; 2015.

[7] Moore, D. B. The Revised ISO 362 Standard for Vehicle Exterior Noise Measurement. Sound and vibration, pp. 19-23, 2006.

[8] Keulen, I. W. V., Duškov, i. M. Inventory study of basic knowledge on tyre/road noise. Ministry of transportation and Waterworks: Rijkswaterstaat, p. 106, 2005. 
[9] National Cooperative Highway Research Program (NCHRP). Acoustic Beamforming: Mapping Sources of Truck Noise. Transportation Research Board: Washington, D. C., p. 80, 2009.

[10] European Commission. Final Report SI2.408210 Tyre/Road Noise: Volume 2, 2001.

[11] Roovers, M.S., Blokland, G.J. Literature study on the Rolling Noise of Truck Tyres. Ministry of transportation and Waterworks: Rijkswaterstaat, p. 92, 2002.

[12] Regulation (EU) No 540/2014 of the European Parliament and of the Council of 16 April 2014 on the sound level of motor vehicles and of replacement silencing systems, and amending Directive 2007/46/EC and repealing Directive 70/157/EEC.

[13] Bosch. Automotive Handbook. 7th ed. Society of Automotive Engineers. Warrendale, 2007.

[14] International Standard Organization (ISO). ISO 10844. Acoustics specification of test tracks for measuring noise emitted by road vehicles and their tyres. London: British Standards Institution, 2011. 